\title{
On the design of automatic voice condition analysis systems. Part II: Review of speaker recognition techniques and study on the effects of different variability factors
}

\author{
J.A. Gómez-García ，L. Moro-Velázquez ， J.I. Godino-Llorente
}

Keywords:

Robust automatic voice condition analysis

Universal background models

Extralinguistic aspects of the speech

Cross-dataset validation

\begin{abstract}
A B S T R A C T
This is the second of a two-part series devoted to the automatic voice condition analysis of voice pathologies, being a direct continuation to the paper on the design of automatic voice condition analysis systems. Part I: review of concepts and an insight to the state of the art. The aim of this study is to examine several variability factors affecting the robustness of systems that automatically detect the presence of voice pathologies by means of audio registers. Multiple experiments are performed to test out the influence of the speech task, extralinguistic aspects (such as sex), the acoustic features and the classifiers in their performance. Some experiments are carried out using state-of-the-art classification methodologies often employed in speaker recognition. In order to evaluate the robustness of the methods, testing is repeated across several corpora with the aim to create a single system integrating the conclusions obtained previously. This system is later tested under cross-dataset scenarios in an attempt to obtain more realistic conclusions. Results identify a reduced subset of relevant features, which are used in a hierarchical-like scenario incorporating information of different speech tasks. In particular, for the experiments carried out using the Saarbrüecken voice dataset, the area under the ROC curve of the system reached 0.88 in an intra-dataset setting and ranged from 0.82 to 0.94 in cross-dataset scenarios. These results let us open a discussion about the suitability of these techniques to be transferred to the clinical setting.
\end{abstract}

\section{Introduction}

Voice impairments arise due to misuse, infections, physiological or psychogenic causes, or due to the presence of other systematic disorders (including neurological), vocal abuse, surgery, trauma, congenital anomalies, irradiation, chemicals affecting vocal folds, etc. [1]. The classical approach to detect voice impairments consists on an instrumental (objective) and perceptual (subjective) evaluation, which are complemented by other types of examinations to determine the existence of a voice disorder and its grade of impairment. In order to assist medical specialists in the diagnosis procedures, a field called automatic voice condition analysis (AVCA) has arisen, providing advantages to traditional detection procedures such as objectiveness or non-invasiveness due to the use of speech signals.
During the first part of this review entitled On the design of automatic voice condition analysis systems. Part I: review of concepts and an insight to the state of the art, some relevant concepts regarding AVCA systems have been described, introducing the most widely employed methodologies that are found in the design of these automatic systems. This second paper explores the design of AVCA systems, carrying out a variety of tests with differing types of speech tasks, types of features and accounting for diverse variability factors. The aim is to design a single generalist AVCA system that is latter tested under cross-dataset scenarios. Some techniques that constitute state-of-the-art in speaker recognition systems and based on the idea of Gaussian Mixture Models (GMM) are also tested out. GMM are generative models that represent the probability density function of a training dataset by means of a linear combination of $G$ multivariate Gaussian components. If the amount of training data is large, it is possible to accomplish a well-trained GMM representing the data; but when it is scarce, other approaches are preferred. In this respect, it is often useful to model, via GMM, a larger auxiliary dataset different to the training dataset. The resulting model is termed Universal Background 


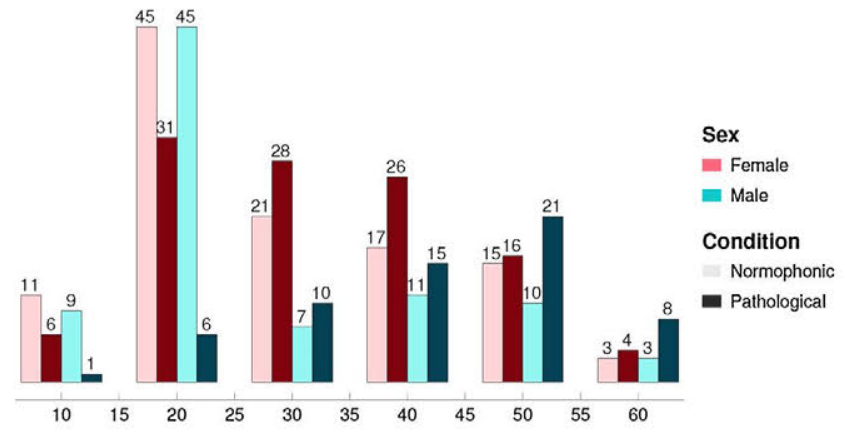

(a) HUPA database

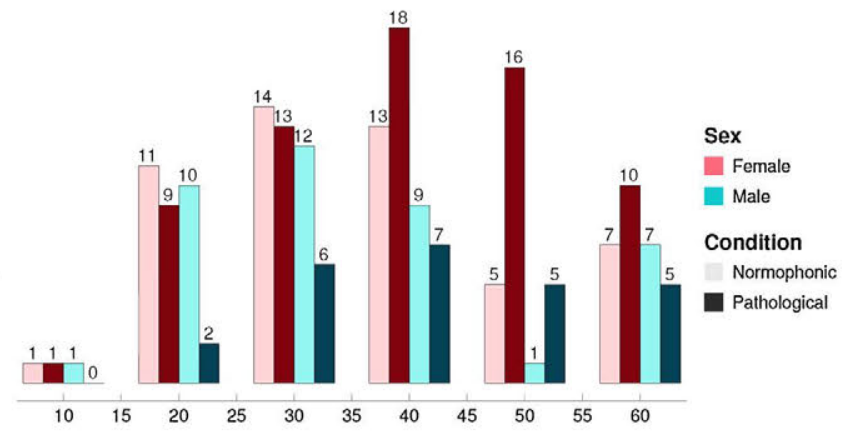

(b) GMar database

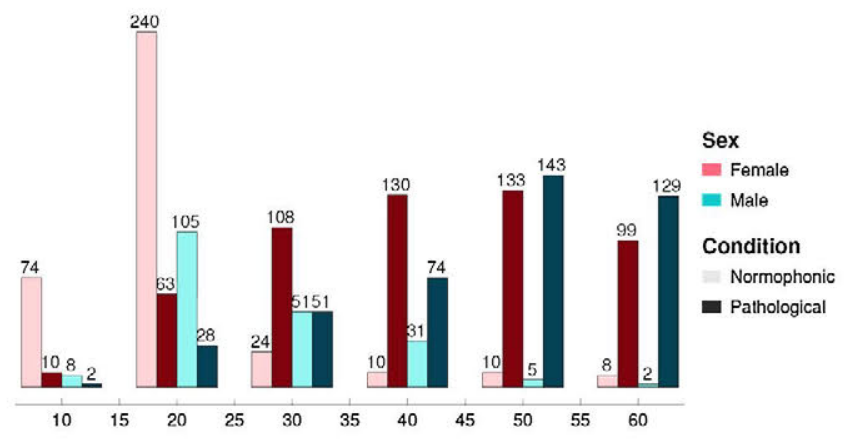

(c) SVD database

Fig. 1. Histograms representing the distribution of patients according to their sex and age for the (a) HUPA, (b) GMar and (c) SVD corpora.

Model (UBM) and serves as an initialisation which is then used to adapt specific - better trained and more generalist - models using the training data. These adapted models are termed GMM-UBM and have been widely employed in several speaker recognition tasks [57]. A variation to GMM-UBM is termed GMM-SVM, which is aimed at combining the discriminatory capabilities of SVM into the GMM framework [53]. Likewise, a further improvement to the GMM-UBM are the $i$-Vectors (IV) [55], which rely on the concept of GMM-UBM and factorial analysis for modelling the training dataset in a total variability space. IV are often accompanied by a Probabilistic Linear Discriminative Analysis (PLDA), which seeks to compensate for the effects of variability factors in the training data [56].

This paper is organised as follows: Section 2 introduces the datasets and the methodological setup of the four major experiments followed in this paper; Section 3 presents the obtained results; Section 4 introduces discussions, whereas Section 5 presents concluding remarks.

\section{Experimental setup}

This section presents the different experimental setups followed throughout the paper. The section begins with a description of the datasets used for training and testing the systems developed, and later presents four methodological frameworks used to test the influence of the speech task, the acoustic features, the classifiers and certain extralinguistic aspects.

\subsection{Acoustic material}

Three datasets containing normophonic and pathological recordings are used as training corpora: Hospital Universitario Príncipe de Asturias (HUPA), Hospital Gregorio Marañón (GMar) and Saarbrücken (SVD) voice disorders corpora. Similarly, four ancillary datasets are also utilised for the construction of the UBM. The ancillary datasets include the EUROM and PhoneDat-I corpora which are composed of normophonic registers of speakers reading passages and pronouncing words, the well-known Massachussets Ear and Eye Infirmary (MEEI) partition of normophonic and dysphonic registers, and the Albayzin dataset which contains recordings of sentences uttered in Spanish. Additionally two extra corpora are used for cross-dataset trials: the Hospital Doctor Negrín dataset (DN) and the Aplicación de las Tecnologías de la Información y las Comunicaciones (ATIC) corpora. A brief description of each one of these datasets is presented next:

\subsubsection{HUPA dataset}

Recorded at the Príncipe de Asturias hospital in Alcalá de Henares, Madrid, Spain [7]. The dataset contains the sustained phonation of the vowel /a/ of 366 adult Spanish speakers (169 pathological and 197 normophonic). Registers have been recorded using the Kay Computerized Speech Lab Analysis station $4300 B$ with a sampling frequency of $50 \mathrm{kHz}$ and 16 bits of resolution. Pathological voices contain a wide variety of organic pathologies including nodules, polyps, oedemas, carcinomas, etc. The distribution of registers according to the sex and age of the speaker is shown in Fig. 1a.

\subsubsection{GMar dataset}

Composed of registers of Spanish speakers phonating the vowels $/ \mathrm{a} /, / \mathrm{i} /$ and $/ \mathrm{u} /$. The dataset has been recorded at the Gregorio Marañón Hospital, Madrid, Spain, using the MediVozCaptura system [6] with a sampling frequency of $22,050 \mathrm{~Hz}$ and 16 bits of resolution. The corpus is composed of 202 audio recordings, from which 95 are of normophonic and 107 of pathological speakers. The distribution of registers for the vowel/a/, according to the sex and age of the speakers is introduced in Fig. 1b.

\subsubsection{SvD dataset}

It holds a collection of audio registers from more than 2000 normophonic and pathological German speakers [8,9]. The dataset was recorded by the Institut für Phonetik at Saarland University and the 
Phoniatry Section of the Caritas Clinic St. Theresia in Saarbrücken, Germany. The corpus comprises recordings of the sustained phonation of vowels /a/, /i/ and /u/ uttered at normal, high and low pitch, as well as with rising-falling pitch. Besides, it incorporates recordings of the sentence Guten Morgen, wie geht es Ihnen? (Good morning, how are you?). Registers have been recorded using a sampling frequency of $50 \mathrm{kHz}$ and 16 bits of resolution. For the purposes of this paper only the vowels $/ \mathrm{a} /, / \mathrm{i} /$ and $/ \mathrm{u} /$ at normal pitch and the running speech recordings have been utilised, having defined a subset of the dataset after removing those registers with a low dynamic range or interferences. After this process, 1538 registers of speakers aged between 16 and 69 years are obtained ( 568 normophonic and 970 pathological). Fig. 1c depicts the distribution according to the sex and age of the speakers in the dataset.

\subsubsection{Ancillary datasets}

As commented above, four ancillary datasets are used in this work. The first, the EUROM corpus, comprises recordings of 60 speakers in each of seven European languages: Danish, Dutch, British English, French, German, Norwegian and Swedish [10]. It has been explicitly designed to aid to the phonetic comparison of languages, with similar materials and recording protocols. In this manner, each corpus has been recorded at $20 \mathrm{kHz}$ and 16 bit of resolution in an anechoic room and balancing the acoustic content among the different languages. In this paper only the German corpus is employed, for which a partition within the corpus called Many Talker set is used. It consists of 63 speakers which are asked to perform two tasks: (i) reading of 100 numbers in the range of 0 to 9999 grouped in 5 blocks of 20 numbers; (ii) reading of 5 sentences coming from 40 texts. The second, the Albayzin dataset, is a Spanish corpora designed for speech recognition purposes [11] and which is composed of three sets: phonetic, application and Lombard speech. Only the phonetic corpus is considered in this work. It contains 6800 recordings of 204 speakers uttering phonetically balanced phrases, which have been digitised using a sampling frequency of $16 \mathrm{kHz}$ and 16 bits of resolution. The third, the PhoneDat-I dataset, contains 200 phonemically balanced artificial German sentences and two readings, namely "the North wind" fable and "a Butter story" [12]. The corpus contains around 20,000 files uttered by $200 \mathrm{Ger}-$ man speakers. All registers have been recorded at a sampling rate of $16 \mathrm{kHz}$ and 16 bit of resolution. Finally, the MEEI voice disorders dataset contains 710 recordings of English speakers, phonating the vowel /a/ and reading the first sentence of the Rainbow Passage [13]. The dataset has been recorded at sampling frequencies ranging from 10 to $50 \mathrm{kHz}$. To ensure a balance in the pathologies under study, a subset of the dataset is chosen in [14]. The resulting partition comprises 226 speakers: 173 pathological and 53 normophonic. The registers have been previously edited to remove the beginning and ending of each utterance, hence omitting the effects of vowel onsets and offsets.

\subsubsection{Corpora for the cross-dataset trials}

Two corpora are considered for cross-dataset trials. On one hand, the DN partition has been recorded by Hospital Dr. Negrín in Las Palmas de Gran Canaria, Spain. It contains 181 registers of Spanish speakers phonating the vowel /a/ [15]. The registers have been recorded at $22,050 \mathrm{~Hz}$ and 16 bits of resolution. A partition of 130 registers has been randomly extracted and used for evaluation purposes.

On the other hand, the ATIC dataset contains recordings of 79 Spanish speakers (58 dysphonic and 21 normophonic) phonating the vowel $/ a /$ [16]. Pathological voices have been obtained from public and private Otorhinolaryngology services in Málaga, Spain, whereas normophonic speakers are recorded from teachers and students recruited at Málaga University. Registers have been recorded in quiet rooms under controlled conditions, digitized at 16 bits and $44,100 \mathrm{~Hz}$.

\subsection{Methodological setup}

Four main experiments are carried out in this paper. The aim is to explore the influence of different variability factors that typically affect the performance of AVCA systems. Additionally, the paper studies the influence on the performance of different classification techniques often employed in the speaker recognition field.

\subsubsection{Experiment 1: variability due to the acoustic material and the feature set}

This experiment contains a series of trials using a variety of feature sets and acoustic material. This allows a direct comparison about the influence of the speech task in AVCA systems, as well as insight about the consistency of the considered features when the acoustic material varies. Likewise, and since no single feature can offer enough discrimination power to completely characterise the properties of dysphonia and normophonia, this allows to study the characteristics rendering the best results in subsequent classification labors. This might potentially permit the design of AVCA systems based on complementary features describing distinct properties of dysphonia.

Trials are performed using all the available acoustic material of the HUPA, SVD and GMar corpora. It is worth noting that not all the acoustic features, which will be mentioned next, can be extracted from running speech, as they rely on conditions - such as stationarity - that cannot be always met. For instance, certain perturbation features depend on the existence of a periodic glottal excitation, assumption that cannot be fulfilled on unvoiced segments of speech. As an alternative to directly analyse running speech, literature often reports procedures based on retrieving voiced segments of speech using voiced-unvoiced algorithms $[17,18]$. This methodology is considered as well in the following trials.

A total of 9 trials are carried out in experiment 1: 1 trial using the HUPA dataset (sustained phonation of the vowel /a/); 3 trials with GMar (sustained phonations of vowels $/ \mathrm{a} /, \mid \mathrm{i} /, / \mathrm{u} /$ ); and 5 with SVD (sustained phonations of vowels $/ \mathrm{a} /, / \mathrm{i} /, \mathrm{u} /$, raw running speech, and voiced segments of the running speech task). The setup followed during each trial is presented in Fig. 2(a) and can be described as follows:

- Preprocessing: all registers are downsampled to $20 \mathrm{kHz}$ (the lowest sampling frequencies of all the available datasets) and max-normalised by dividing the signal by its absolute largest value. Then, a framing and a Hamming windowing procedure is followed, where the length of the window is determined depending on the feature sets that are extracted, and the overlap is varied to ensure that all the sets of characteristics contain the same number of frames.

- Characterisation: this stage has the goal of extracting features capable of portraying the properties of normophonic and dysphonic conditions. The idea is to extract for each windowed frame, a $d$-dimensional vector of characteristics, $\vec{x}=\{x[1], \cdots, x[d]\}$ which is associated to a label $\ell$, describing properties of the segment. The sets of characteristics that are considered in this paper are descriptors of vocal quality. These are illustrated in Fig. 2(b) and are described next:

Perturbation features (Pert set): measure the presence of additive noise resulting from an incomplete glottal closure of the vocal folds, and the presence of modulation noise which is the result of irregularities in the movements of the vocal folds. Three perturbation metrics are employed: Normalised Noise 


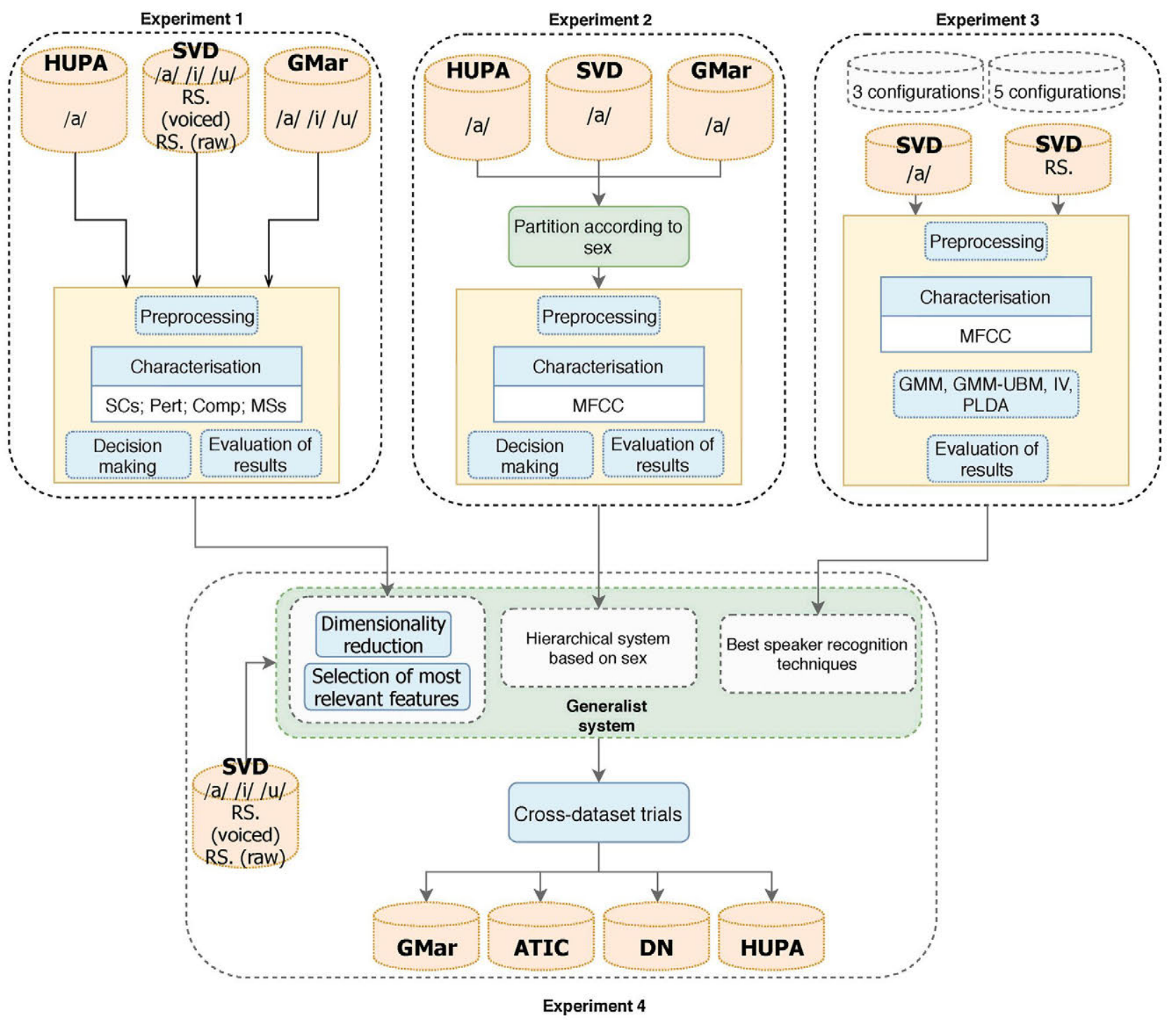

(a) Methodological stages of the AVCA system.

\begin{tabular}{lllllll}
\hline Set & Subset & \multicolumn{5}{l}{ Features } \\
\hline Pert & - & NNE & CHNR & GNE & \\
\hline SCs & - & PLP & MFCC & CPPS & LHr & \\
\hline MSs & - & MSH & CIL & RALA & MSP & \\
\hline & Dyn & D2 & LLE & RPDE & & \\
Comp & LR & He & DFA & & & \\
& Reg & ApEn & SampEn & mSampEn & GSampEn & FuzzyEn \\
& Ent & rHMMEn & rHMMEn & FuzzyEn & & \\
\hline
\end{tabular}

(b) Feature sets and subsets used for characterisation.

Fig. 2. (a) Methodology followed in this paper for the design of the AVQA system; (b) table containing all the considered sets of features. 
Entropy (NNE) [19], Cepstral Harmonics-to-Noise Ratio (CHNR) [20] and Glottal-to-Noise Excitation Ratio (GNE) [21].

Spectral and cepstral features (SCS set): measure the harmonic components of the voice. This set of features encloses Perceptual Linear Prediction coefficients (PLP) [22], Mel-Frequency Cepstral Coefficients (MFCC) [23], Smoothed Cepstral Peak Prominence (CPPS) [24] and Low-to-High Frequency Spectral Energy Ratio (LHr) [5]. The number of PLP and MFCC coefficients are varied in the range $[10,20]$ with steps of 2 .

Features based on modulation spectrum (MSs set): rely on the computation of the modulation spectrum, that characterises the modulation and acoustic frequencies of input voices [25]. The features considered in the set are: Modulation Spectrum Homogeneity (MSH), Cumulative Intersection Point (CIL), Rate of Points above Linear Average (RALA) and Modulation Spectrum Percentile (MSP) $m$, where the sub-index is referred to the percentile that is used, i.e. $\mathrm{MSP}_{25}, \mathrm{MSP}_{75}$ and $\mathrm{MSP}_{95}[26,38]$.

Complexity (Comp set): this family of parameters characterises the dynamics of the system and its structure. Several features are extracted, which are further grouped according to the properties they measure. Hence: (i) the first subset comprises dynamic invariants (Dyn subset) extracted from a reconstructed attractor such as the Correlation Dimension (D2), the Largest Lyapunov Exponent (LLE) [27], and the Recurrence Period Density Entropy (RPDE) [28]; (ii) the second subset contains features which measure long-range correlations ( $L R$ subset), such as Hurst Exponent (He) and Detrended Fluctuation Analysis (DFA) [29,28]; (iii) the third subset includes regularity estimators (Reg subset) which are based on entropylike quantifiers. It encompasses Approximate Entropy (ApEn) [30], Sample Entropy (SampEn) [31], Modified Sample Entropy (mSampEn) [32], Gaussian Kernel Sample Entropy (GSampEn) [33] and Fuzzy Entropy (FuzzyEn) [34]; (iv) finally, the fourth subset includes entropy estimators (Ent subset) such as Permutation Entropy (PE) [35,36], Rényi Hidden Markov Model Entropy (rHMMEn) and Shannon Hidden Markov Model Entropy (sHMMEn) $[23,37]$.

For the trials based on sustained phonation, Hamming windows of $40 \mathrm{~ms}$ are employed for the Pert and SCs sets to ensure that each frame contains at least one pitch period, whereas windows of $55 \mathrm{~ms}$ length are used in the Comp sets as suggested in [23]. Likewise, for experiments in the MSs set, segments of $180 \mathrm{~ms}$ are utilised as in [26,38].

For those trials involving running speech, two different scenarios are considered. In the first, only segments representing voice are extracted from the speech. These segments are extracted automatically by means of the MAUS software, as described in [42]. The second makes use of the raw running speech registers, but using window lengths in the range $[10,30] \mathrm{ms}$ with $5 \mathrm{~ms}$ steps. These values are selected to avoid violating the stationarity assumptions. A voice activity detector has been also employed to reject unwanted segments [54].

- Decision making: Given a training set of observations $\boldsymbol{X}=\left\{\vec{x}_{1}, \cdots, \vec{x}_{n}, \cdots, \vec{x}_{N}\right\}$, associated to a label vector $\vec{\ell}=$ $\left\{\ell_{1}, \cdots, \ell_{n}, \cdots, \ell_{N}\right\}$, the aim of the decision making procedure is to learn a mapping from the input observations to the labels. GMM classifiers are used for modelling class membership before taking decisions using log-likelihood functions.

- Evaluation of results: The Area Under the ROC curve (AUC) is the preferred measure of performance evaluation. A $k$-folds crossvalidation procedure has been employed setting the number of folds to $k=11$. Detection-Error Tradeoff (DET) curves and standard performance metrics for a binary classifier are considered for each one of the aforementioned sets of features: Accuracy (ACC), Sensitivity (SE), Specificity (SP).

\subsubsection{Experiment 2: design of a hierarchical system based on the} sex of the speaker

The effects of the extralinguistic trait of sex in the design of AVCA systems are explored. The interest of this approach in AVCA is in the possibility of constructing hierarchical-like schemes taking into account the variability introduced by certain extralinguistic factors separately. The idea is to segment the population according to extralinguistic characteristics to train more accurate models. As a result, the complexity of the voice pathology detection task is decreased, simpler signal processing models are generated, and a subsequent performance improvement is expected. To analyse the impact of the sex of the speakers in detection tasks, a sex-independent and a sex-dependent system (an AVCA for female speakers and another for males) are designed.

Three trials are carried out using the partitions of the vowel /a/ belonging to the HUPA, SVD and GMar dataset. The setup followed for all the trials is presented in Fig. 2(a), whereas the main stages are described next:

1. Preprocessing: all registers are downsampled to $20 \mathrm{kHz}$ and maxnormalised. For the sex-dependent systems the datasets are decomposed according to sex of the speaker.

2. Characterisation: Only MFCC features are extracted in the current experiment. The number of MFCC coefficients is varied in the range $[10,20]$ with steps of 2 . Hamming windows of $40 \mathrm{~ms}$ with a $50 \%$ of overlapping between consecutive frames are employed.

3. Classification: decision making is carried out using a GMM classifier, varying the number of Gaussian components as $\left\{2^{i}\right\}: i \in$ $\mathbb{Z} ; 1 \leq i \leq 9$.

4. Evaluation of results: the same metrics used in the experiment 1 are considered.

\subsubsection{Experiment 3: testing out the performance of classification} techniques used in speaker recognition

The performance of classifiers relying on the idea of UBMs in conjunction with MFCC features, a setup that has been proven useful in speaker recognition tasks - but that has not been widely explored in AVCA systems - is studied. In this experiment only the registers of the vowel $/ \mathrm{a} /$ and running speech belonging to the SVD partition are examined, thus defining two trials. In the one using sustained phonation, three configurations are defined in accordance to the auxiliary datasets that are used to train the UBM and compensation models: (i) configuration $C_{1}$ employs normophonic registers of HUPA corpora; (ii) configuration $C_{2}$ uses normophonic and dysphonic recordings of HUPA, MEEI and GMar (vowel /a/ only); (iii) configuration $C_{3}$ employs normophonic data of HUPA, MEEI, GMar and vowels extracted from EURoM to compare the influence of using acoustic material coming from different speech tasks (running speech, sustained vowels /a/,/i/ or / / /). With respect to EUROM, vowels are used to match as much as possible the acoustic content of the SVD partition. Since there is access to the phonological transcription of the audio files, these segments are extracted automatically by means of the MAUS software, as described in [42].

For the trial using running speech, diverse ancillary datasets are employed for training the UBM and compensation models: (i) configuration $C_{1}$ : employs normophonic and dysphonic recordings of the vowels $/ \mathrm{a} /, / \mathrm{i} /, / \mathrm{u} /$ of the HUPA and GMar datasets, plus normophonic vowels extracted from the EUROM and Albayzin corpora; (ii) configuration $C_{2}$ : considers normophonic-only recordings of the vowels /a/, /i/, / u/ of the HUPA and GMar datasets, plus normophonic vowels extracted from the EUROM and Albayz in corpora; (iii) configuration $C_{3}$ : characterises the normophonic sentences of MEEI, EUROM and Albayzin; (iv) configuration $\mathrm{C}_{4}$ : characterises the normophonic sentences of EUROM; ( $v$ ) configuration $C_{5}$ : characterises the normophonic sentences of EUROM and PhoneDat-I. 
Table 1

Tested configurations for training the UBM models in experiment 3.

\begin{tabular}{|c|c|c|c|c|}
\hline & Configuration & Datasets & Speech tasks & Content \\
\hline \multirow[t]{3}{*}{ Sustained phonation } & $C_{1}$ & HUPA & $|a|$ & No. \\
\hline & $C_{2}$ & HUPA, MEEI, GMar & $\mid \mathrm{a} /$ & No. + Dy. \\
\hline & $C_{3}$ & HUPA, MEEI, GMar, EUROM & $|\mathrm{a}|+|\mathrm{i}|+\mid \mathrm{u} /+\mathrm{RSv}$ & No. \\
\hline \multirow[t]{5}{*}{ Running speech } & $C_{1}$ & HUPA, GMar, EUROM, Albayzin & $|\mathrm{a}|+|\mathrm{i}|+|\mathrm{u}|+\mathrm{RSv}$ & No. + Dy. \\
\hline & $C_{2}$ & HUPA, GMar, EUROM, Albayzin & $|\mathrm{a}|+|\mathrm{i}|+|\mathrm{u}|+\mathrm{RSv}$ & No. \\
\hline & $C_{3}$ & MEEI, BUROM, Albayzin & RSr. & No. \\
\hline & $C_{4}$ & EUROM & RSr. & No. \\
\hline & $C_{5}$ & EUROM, PhoneDat-I & RSr. & No. \\
\hline
\end{tabular}

RSr.: raw running speech; RSv.: vowels extracted from running speech; No.: normophonic; Dy.: dysphonic.

Table 1 summarises the trials and the configurations in experiment 3 , whereas the experimental setup is introduced graphically in Fig. 2(a) and described as follows:

1. Preprocessing: for the trial involving sustained phonation, $40 \mathrm{~ms}$ Hamming windows with a $50 \%$ of overlapping between consecutive frames are employed, whereas for the running speech experiments, the length of the window is defined according to the best outcomes of experiment 2 (where lengths were varied in the range $[10,30] \mathrm{ms}$ with steps of $5 \mathrm{~ms}$ ).

2. Characterisation: MFCC features are extracted from each one of the frames obtained in the preprocessing stage. The number of MFCC coefficients is varied between $[10,20]$ with steps of 2 coefficients.

3. Decision machines: GMM, GMM-UBM, IV, PLDA and GMM-SVM classifiers are employed. The number of Gaussian components is varied in such a manner that $\left\{2^{i}\right\}: i \in \mathbb{Z} ; 1 \leq i \leq 9$.

4. Evaluation of results: the same procedure followed in previous experiments is carried out.

2.2.4. Experiment 4: combination of the best systems.

This experiment is built around the lessons learnt during the first three experiments. Presumably it is to be of a hierarchical type, it will use a subset of informative features, and will employ all the available acoustic material to provide a single decision about the condition of speakers.

Two trials are considered in the current experiment: (i) One which provides a single decision about the condition of patients by combining the results of the systems based on the vowels /a/, /i/, /u/ and the running speech task of the SVD dataset; (ii) other designed to test the capabilities of the system in a cross-dataset scenario. In particular, the system is assessed using the HUPA, ATIC, DN and GMar corpora.

The methodological stages that are followed in both trials are presented in Fig. 2(a) and are described next:

1. Preprocessing: all registers are downsampled to $20 \mathrm{kHz}$ and maxnormalised for the trials involving sustained phonation, $40 \mathrm{~ms}$ Hamming windows with a $50 \%$ of overlapping are used. For those using running speech, the length of the window is defined according to the outcomes of experiment 1.

2. Characterisation: two systems are considered. The first is designed with those features providing the best results in experiment 1 . To this end, three dimensionality reduction methods are employed to rank features from the most to the least relevant, in the search for a decrease in the computational burden, an improvement in the accuracy of the analyses, and the avoidance of problems such as the curse of dimensionality [40]. This analysis is performed independently for the HUPA, SVD and GMar datasets by means of three selection techniques [41]: Maximal Information Maximization (MIM), Minimal Redundancy Maximal Relevance (mRMR) and Joint Mutual Information (JMI).
To generalize results across datasets, acoustic material and features, a scoring procedure is now performed. In this manner and with the results of the ranking techniques and for a certain dataset, the scoring procedure rewards the best features with a low score, while penalizing the worst with a large value. These scores are then summed up across datasets and feature selection techniques. At the end, the features with the lowest scores are regarded as the most informative and consistent and are employed for further testing. In addition to this system, another is designed with MFCC features as it is to be used for the speaker recognition classification techniques. If the hierarchical system is build up (in accordance to results of experiment 2), the number of MFCC coefficients is varied in the range $[10,20]$ with steps of 2 , otherwise, the parameter that render the best results in experiment 1 is used.

3. Decision machines: GMM classifiers are used to train the system employing the most consistent set of features. The MFCC system utilizes the classifiers that render the highest efficiency as given by the results of experiment 3 . The number of Gaussian components is varied in such a manner that $\left\{2^{i}\right\}: i \in \mathbb{Z} ; 1 \leq i \leq 9$.

4. Fusion of results: logistic regression is employed to fuse the system using the most consistent features and a GMM classifier, and the system based on MFCC and classification based on UBM. A further fusion is considered to combine the information coming from diverse speech tasks, and thus, to provide a single decision about the condition of speakers.

5. Evaluation of results: the same evaluation procedures followed in previous experiments is carried out.

\section{Results}

\subsection{Experiment 1: variability due to the acoustic material and the type of features}

In total 9 trials are performed: one using the vowel /a/ in HUPA; three for the vowels /a/, /i/, /u/ in GMar; and three for the vowels $|\mathrm{a} /,| \mathrm{i} /, \mid \mathrm{u} /$, and two using running speech in the SVD corpus.

\subsubsection{HUPA dataset}

The performance metrics of all the considered sets of features and the DET curves of the best performing subsets, using the vowel $\mid a /$ of the HUPA dataset are presented in Fig. 3.

From the obtained outcomes it can be observed that the best results - in terms of AUC - are provided by the Pert set (0.85), whereas in the SCs set, MFCC and PLP achieve an almost equivalent performance (AUC of 0.80 and 0.79 respectively). Likewise, the subset Ent achieves the best results within the Comp set (0.83) while LR provides the worst $(0.60)$. 


\begin{tabular}{llllll}
\hline Set & Subset & ACC & SP & SP & AUC \\
\hline Pert & - & $76.61 \pm 4.30$ & 0.77 & 0.77 & 0.85 \\
\hline MSs & - & $71.77 \pm 4.57$ & 0.74 & 0.70 & 0.79 \\
\hline \multirow{4}{*}{ SCs } & CPPS+LHr & $62.10 \pm 4.93$ & 0.60 & 0.64 & 0.69 \\
& MFCC & $69.62 \pm 4.67$ & 0.66 & 0.74 & 0.79 \\
& PLP & $66.94 \pm 4.78$ & 0.58 & 0.77 & 0.80 \\
\hline \multirow{4}{*}{ Comp } & LR & $56.18 \pm 5.04$ & 0.51 & 0.62 & 0.60 \\
& Dyn & $65.59 \pm 4.83$ & 0.63 & 0.68 & 0.75 \\
& Reg & $69.89 \pm 4.66$ & 0.68 & 0.72 & 0.78 \\
& Ent & $75.00 \pm 4.40$ & 0.75 & 0.75 & 0.83 \\
\hline
\end{tabular}

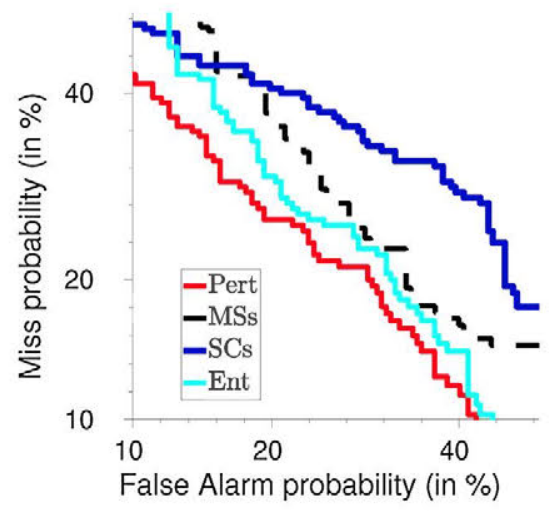

Fig. 3. Experiment 1: results for the vowel/a/ in the HUPA database.

\subsubsection{GMar dataset:}

The performance metrics of all the considered sets of features and the DET curves of the best performing subsets, for the vowels /a/, /i/ and /u/ in the GMar dataset are presented in Fig. 4.

Respecting the vowel /a/, the best outcomes arise when using the Ent subset in Comp (AUC=0.83). Similarly, MSs, Pert and the whole SCs set provide acceptable outcomes, with AUC ranging from 0.73 to 0.77 . By contrast, and for the vowel / i/, MSs achieves the best results (0.76), followed by the Pert set and the Reg subset, which accomplish in both cases an AUC of 0.73. Finally, and when the vowel $/ \mathrm{u} /$ is studied, the best results are given by the LR and the Ent subsets (AUC of 0.74 and 0.73 respectively). Notwithstanding, the PLP subset and the MSs set provide comparable results ( 0.73 and 0.72 respectively).

\subsubsection{SVD dataset}

The performance metrics of all the considered sets of features and the DET curves of the best performing subsets, for the vowels /a/, /i/ and /u/ are presented in Fig. 5a.

Considering the vowel $/ \mathrm{a} /$, the best efficiency arises with the Pert set (AUC $=0.78$ ), followed by MFCC and PLP whose performance is alike ( 0.77 and 0.76 respectively). Within the Comp set, Ent and Reg

\begin{tabular}{llllll} 
& & \multicolumn{5}{c}{ Vowel /a/ } \\
\hline Set & Subset & ACC & SP & SE & AUC \\
\hline Pert & - & $65.35 \pm 6.56$ & 0.65 & 0.65 & 0.77 \\
\hline MSs & - & $67.82 \pm 6.44$ & 0.65 & 0.70 & 0.76 \\
\hline \multirow{4}{*}{ SCs } & CPPS+LHr & $66.83 \pm 6.49$ & 0.67 & 0.66 & 0.73 \\
& MFCC & $69.31 \pm 6.36$ & 0.69 & 0.69 & 0.77 \\
& PLP & $68.81 \pm 6.39$ & 0.67 & 0.70 & 0.76 \\
\hline \multirow{4}{*}{ Comp } & LR & $56.18 \pm 5.04$ & 0.51 & 0.62 & 0.60 \\
& Dyn & $65.59 \pm 4.83$ & 0.63 & 0.68 & 0.75 \\
& Reg & $69.89 \pm 4.66$ & 0.68 & 0.72 & 0.78 \\
& Ent & $75.00 \pm 4.40$ & 0.75 & 0.75 & 0.83 \\
\hline & & & & &
\end{tabular}

\begin{tabular}{lccc}
\hline \multicolumn{5}{c}{ Vowel /i/ } \\
\hline ACC & SP & SE & AUC \\
\hline $66.32 \pm 6.72$ & 0.65 & 0.68 & 0.73 \\
\hline $67.82 \pm 6.44$ & 0.65 & 0.70 & 0.76 \\
\hline $60.53 \pm 6.95$ & 0.62 & 0.59 & 0.67 \\
$60.53 \pm 6.95$ & 0.61 & 0.60 & 0.65 \\
$58.42 \pm 7.01$ & 0.60 & 0.57 & 0.65 \\
\hline $61.05 \pm 6.93$ & 0.59 & 0.64 & 0.65 \\
$64.74 \pm 6.79$ & 0.63 & 0.67 & 0.70 \\
$61.58 \pm 6.92$ & 0.48 & 0.75 & 0.73 \\
$60.53 \pm 6.95$ & 0.61 & 0.60 & 0.68 \\
\hline
\end{tabular}

\begin{tabular}{llll}
\hline \multicolumn{4}{c}{ Vowel /u/ } \\
\hline ACC & SP & SE & AUC \\
\hline $61.36 \pm 7.19$ & 0.64 & 0.59 & 0.70 \\
\hline $63.64 \pm 7.11$ & 0.63 & 0.64 & 0.72 \\
\hline $59.09 \pm 7.26$ & 0.58 & 0.60 & 0.68 \\
$62.50 \pm 7.15$ & 0.62 & 0.63 & 0.68 \\
$67.05 \pm 6.94$ & 0.70 & 0.64 & 0.73 \\
\hline $61.36 \pm 7.19$ & 0.64 & 0.59 & 0.74 \\
$58.52 \pm 7.28$ & 0.52 & 0.64 & 0.70 \\
$63.07 \pm 7.13$ & 0.60 & 0.66 & 0.68 \\
$63.07 \pm 7.13$ & 0.67 & 0.59 & 0.73 \\
\hline
\end{tabular}
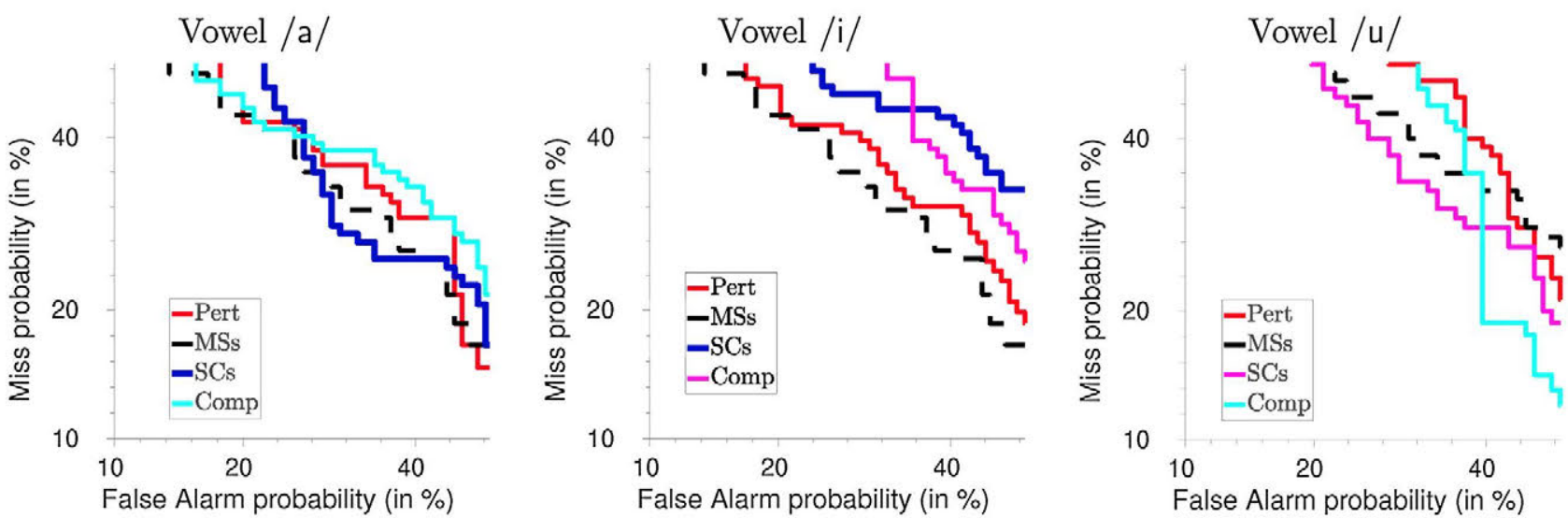

Fig. 4. Experiment 1: results for the vowels $/ \mathrm{a} /, \mathrm{i} /$ and $/ \mathrm{u} /$ in the GMar database. 


\begin{tabular}{llllll}
\cline { 3 - 5 } Set & Subset & ACC & SP & SE & AUC \\
\hline Pert & - & $68.79 \pm 2.32$ & 0.68 & 0.69 & 0.78 \\
\hline MSs & - & $66.67 \pm 2.35$ & 0.60 & 0.70 & 0.73 \\
\hline \multirow{4}{*}{ SCs } & CPPS+LHr & $61.12 \pm 2.44$ & 0.61 & 0.61 & 0.68 \\
& MFCC & $70.48 \pm 2.28$ & 0.67 & 0.73 & 0.77 \\
& PLP & $71.07 \pm 2.27$ & 0.70 & 0.72 & 0.77 \\
\hline \multirow{3}{*}{ Comp } & D. & $61.31 \pm 2.43$ & 0.59 & 0.63 & 0.68 \\
& Reg & $63.78 \pm 2.40$ & 0.62 & 0.65 & 0.73 \\
& Ent & $67.75 \pm 2.34$ & 0.55 & 0.75 & 0.74 \\
\hline
\end{tabular}

\begin{tabular}{llll}
\hline \multicolumn{4}{c}{ Vowel $/ i /$} \\
\hline ACC & SP & SE & AUC \\
\hline $64.37 \pm 2.39$ & 0.64 & 0.65 & 0.72 \\
\hline $63.20 \pm 2.41$ & 0.64 & 0.63 & 0.70 \\
\hline $57.87 \pm 2.47$ & 0.55 & 0.59 & 0.62 \\
$68.73 \pm 2.32$ & 0.67 & 0.69 & 0.76 \\
$68.21 \pm 2.33$ & 0.67 & 0.69 & 0.75 \\
\hline $59.04 \pm 2.46$ & 0.57 & 0.60 & 0.61 \\
$62.29 \pm 2.42$ & 0.62 & 0.63 & 0.68 \\
$63.46 \pm 2.41$ & 0.62 & 0.65 & 0.69 \\
$61.25 \pm 2.43$ & 0.60 & 0.62 & 0.65 \\
\hline
\end{tabular}

\begin{tabular}{llll}
\hline \multicolumn{5}{c}{ Vowel $/ \mathrm{u} /$} \\
\hline $\mathrm{ACC}$ & $\mathrm{SP}$ & $\mathrm{SE}$ & $\mathrm{AUC}$ \\
\hline $59.88 \pm 2.45$ & 0.59 & 0.60 & 0.66 \\
\hline $61.05 \pm 2.44$ & 0.53 & 0.66 & 0.67 \\
\hline $58.13 \pm 2.47$ & 0.55 & 0.60 & 0.63 \\
$65.41 \pm 2.38$ & 0.64 & 0.66 & 0.71 \\
$64.69 \pm 2.39$ & 0.62 & 0.66 & 0.71 \\
\hline $58.06 \pm 2.47$ & 0.57 & 0.59 & 0.64 \\
$61.51 \pm 2.43$ & 0.58 & 0.64 & 0.68 \\
$63.78 \pm 2.40$ & 0.50 & 0.72 & 0.67 \\
$59.95 \pm 2.45$ & 0.57 & 0.62 & 0.66 \\
\hline
\end{tabular}
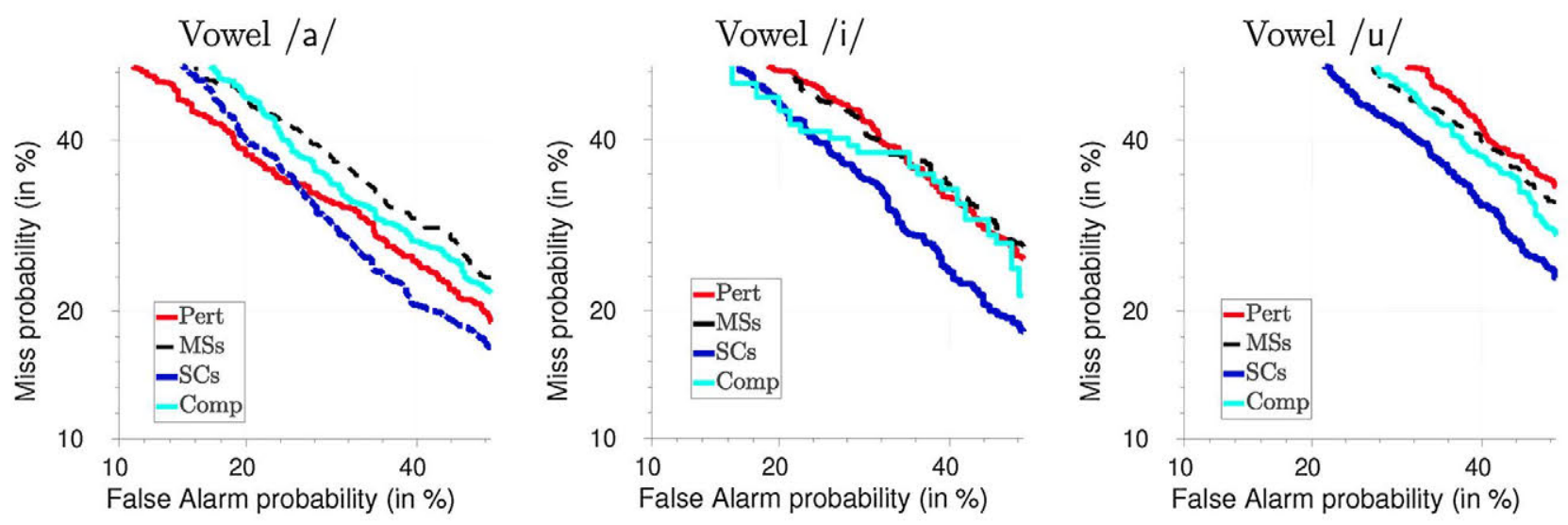

a. Results for vowels /a/, /i/ and /u/

\begin{tabular}{llllll} 
& & \multicolumn{4}{c}{ Running speech - raw speech } \\
\hline Set & Length & ACC & SP & SE & AUC \\
\hline CPPS+LHr & $25 \mathrm{~ms}$ & $62.26 \pm 2.46$ & 0.60 & 0.64 & 0.67 \\
MFCC (18) & $20 \mathrm{~ms}$ & $80.32 \pm 2.02$ & 0.74 & 0.84 & 0.86 \\
PLP (18) & $20 \mathrm{~ms}$ & $77.90 \pm 2.11$ & 0.65 & 0.85 & 0.85 \\
\hline
\end{tabular}

\begin{tabular}{lllll} 
& \multicolumn{4}{l}{ Running speech - extracted vowels } \\
\cline { 2 - 5 } Set & ACC & SP & SE & AUC \\
\hline Pert & $66.69 \pm 2.39$ & 0.67 & 0.67 & 0.74 \\
CPPS+LHr & $56.15 \pm 2.52$ & 0.52 & 0.58 & 0.58 \\
MFCC (16) & $76.96 \pm 2.14$ & 0.70 & 0.81 & 0.84 \\
PLP (18) & $75.55 \pm 2.18$ & 0.67 & 0.80 & 0.82 \\
\hline
\end{tabular}

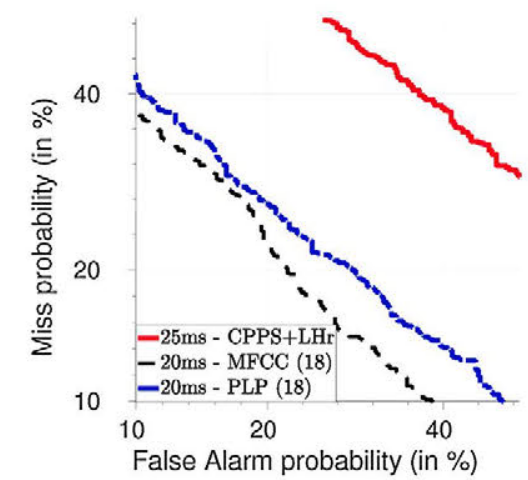

b. Results using the raw running speech task.

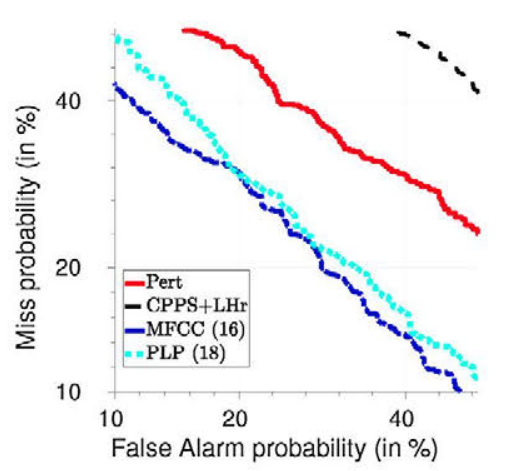

c. Vowels extracted from running speech.

Fig. 5. Experiment 1: results for the SVD database using (a) the vowels /a/,/i/and /u/; (b) the raw running speech task; (c) vowels extracted from the running speech task. 


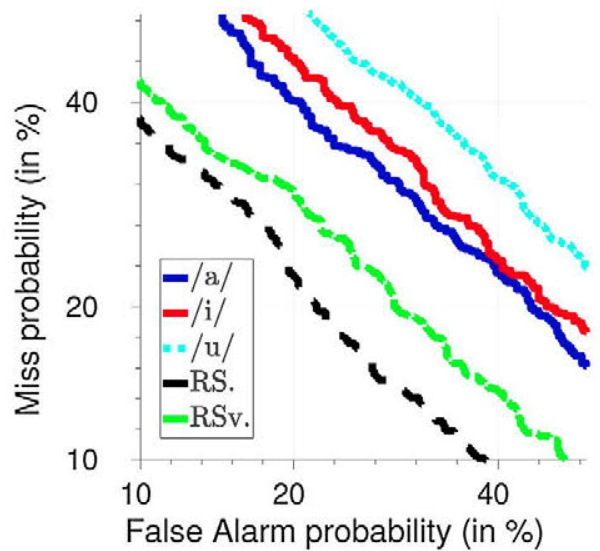

(a) DET curve for the SVD dataset.

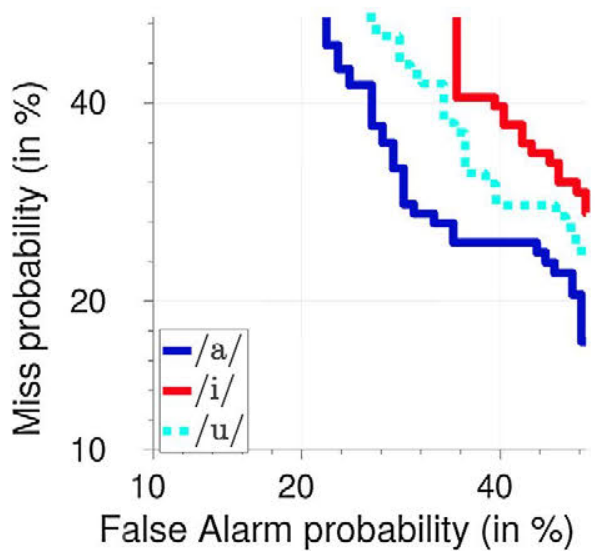

(b) DET curve for the GMar dataset.

Fig. 6. Experiment 1 DET curves using MFCC features and speech material of different nature belonging to (a) sVD dataset; and (b) GMar dataset. RS.: running speech; RSv.: vowels extracted from running speech.

provides the best performance ( 0.75 and 0.74 respectively). With regards to the vowel /i/, MFCC and PLP features accomplish the best results in the trial (AUC of 0.76 and 0.75 respectively), followed by other good performing sets such as Pert and MSs (AUC of 0.72 and 0.70 respectively). In a similar fashion, the results for the vowel $/ \mathrm{u} /$ crown MFCC and PLP as one of the best performing features when vowels are considered, but closely followed by the remaining.

Fig. 5b presents the performance metrics of all the considered sets of features and the DET curves of the best performing subsets in the SVD dataset, for the running speech task using the raw acoustic material; whereas Fig. 5c introduces the results when using vowels extracted from the running speech registers.

With regards to the trial using the raw speech, it is observed that both PLP and MFCC features perform almost equivalently ( 0.85 for PLP and 0.86 for MFCC). In a similar way, the window length that achieves the best results is in this case of $20 \mathrm{~ms}$.

Respecting the trial using extracted vowels from running speech, it is worth noting that it was not possible to employ the same characterisation stage as in when using sustained phonation, as there are some practical reasons that difficult this type of analysis. In particular, the window length that is employed in some sets of features is too long to allow the extraction of characteristics. This is expected, as running speech is composed of fast transitions between silence, voiced or unvoiced sounds and intervals of sustained phonation are not produced frequently. Indeed, for characterisation with the MSs set it is necessary to include voiced segments with a window size of $180 \mathrm{~ms}$. However, there are not vocal segments with such length in the SvD dataset. This behaviour is also encountered when analyzing complexity features, where the number of segments whose length is $55 \mathrm{~ms}$ is greatly restricted. For this reason, the reported results only employ sets using windows of $40 \mathrm{~ms}$, i.e., Pert and SCs to characterise voiced segments of speech. The outcomes indicate that the MFCC features provide the best results (AUC $=0.86)$ next to PLP $(A U C=0.85)$. In comparison with the outcomes of the running speech trial, there is a light decrease of performance when using the vowels extracted from the speech.

\subsubsection{General comments}

During this experiment several sets of features have been employed for detection tasks, using different datasets of sustained vowels and running speech. By virtue of the obtained results, we can infer that there is no single feature or set of features that always perform better than the others. Indeed, depending on the type of acoustic material or the particularities of the corpus, a certain set of features outperforms the others. Despite that, there are some interesting observations indicating tendencies of good performance. For instance, Pert has proven to be useful for detection purposes as ascertained by the large AUC values that varied from 0.66 using the vowel $/ \mathrm{u} /$ and SVD, to 0.85 for the HUPA dataset (or to 0.86 if running speech is considered). In a similar fashion, the SCs set provides large AUC values that ranged from 0.71 for the vowel $/ \mathrm{u} /$ and the SVD dataset, to 0.8 for the HUPA dataset. In most of the cases (except for the vowel /i/ of the GMar dataset), either PLP or MFCC outperformed the results of CPPS+LHr. Regarding the MSs set, the performance of the whole subset remains among the highest compared to the remaining sets, with an AUC that varies from 0.67 for the vowel $/ \mathrm{u} /$ and the SVD corpus, to 0.79 using HUPA. Finally, and respecting the complexity features, the results indicate that the subset providing the worst general performance is LR as in almost all cases (but for GMar and vowel $/ \mathrm{u} /$ ), it is surpassed by the remaining subsets. Similarly, the performance of Reg and Ent tends to be superior to that of Dyn.

In reference to the acoustic material employed, some trends are observed as well. Revisiting the results of the trials using sustained phonation, it can be inferred that the vowel /a/ achieves, in all cases, better results than vowels $/ \mathrm{i} /$ and $/ \mathrm{u} /$, no matter the set that is analysed. When including running speech into the analysis, it is found that the spectral/cepstral features are in general better when the sentence is employed in comparison to the use of a sustained phonation. By contrast, for the Pert set, the vowel /a/ provides a better performance than when using running speech. In an attempt to compare speech material of different nature with the same set of features, Fig. 6 presents the best DET curves for all the trials involving the SVD and GMar corpora. MFCC features are depicted as they are calculated in all tests, including those based on running speech. The outcomes suggest that - despite the difficulties of comparing results - there is a certain tendency to favour the employment of acoustic material based on running speech when spectral/cepstral analysis is considered. In addition, in both cases the vowel /a/ presents a better performance in comparison to using vowels $/ \mathrm{i} /$ or $/ \mathrm{u} /$.

It is important to highlight that a fair comparison between speech tasks is difficult, as there are certain differences in the amount of acoustic material that is available when speech and voice are compared. As a matter of example, and taking the SVD dataset, the trial using the raw running speech material includes 219.000 frames into the analysis, whereas 36.691 voiced frames are 
encountered when including only vowels extracted from speech. By contrast, the analysis of the sustained phonation of the vowel /a/ is conformed by 95.422 frames.

In a direct comparison of the two detection tasks using running speech, it is found that better results are obtained when no voiced detector is included. This might be simply a consequence of having used an automatic system for the segmentation of the voice recordings, as some errors might be introduced during this process, specially when pathological voice is considered. In any case, disregarding the use of voiced detectors comes with the added benefit of reducing the complexity of the AVCA system, at expenses of having to process irrelevant information.

\subsection{Experiment 2: effects of extralinguistic traits}

Fig. 7 introduces the performance metrics of the sexindependent and sex-dependent systems trained for each one of the employed datasets.

Results indicate that in the HUPA dataset, there is a tendency to a performance enhancement when the sex of the speaker is accounted in the classification ( $A U C=0.81$ vs. $A U C=0.79$ ). This is expected since modelling female and male systems separately lead to a larger AUC ( 0.85 and 0.81 respectively) compared to a sexindependent scenario (0.79). The outcomes of the trial involving SVD also suggest that, in comparison to a sex-independent system, there is a certain tendency towards an efficiency improvement when the sex is deemed in the design of AVCA systems (AUC $=0.78$ when the sex is considered vs. AUC $=0.77$ when it is not). Unlike the previous trial, the female-only models achieve higher detection rates than the ones provided by the male-only models (AUC $=0.79$ vs. AUC $=0.77$ ). In the case of GMar, the same tendency of the previous two trials is observed, i.e., the model considering the sex of the speakers outperforms the sex-independent system ( $A U C=0.78$ vs. AUC $=0.77$ ). Just as with the HUPA dataset, the results of the maleonly models outperform those of the female-only ones ( $A U C=0.82$ and $\mathrm{AUC}=0.77$ respectively).

Despite the simplicity of the trials, some interesting observations arise. On one hand, and respecting the inclusion of sex information, the outcomes indicate that accounting for the speaker's sex improves the efficiency in pathology detection tasks. Indeed, in the three datasets there is an absolute performance improvement which varied between $4 \%$ (using HUPA) to $0.3 \%$ (using SVD) compared to the sex-independent detector. It is worth noting that, in general, the best sex-independent and the best female/male detection systems establish a distinct number of MFCC coefficients. Ultimately, this may be a symptom of having decomposed the problem according to the speaker's sex that induce to different "optimal" operation points, i.e., the number of coefficients maximising performance for female models is not necessarily the same for male ones or when both sexes are considered in conjunction. This might be explained by the significant differences in the vocal tract and vocal folds of male and female speakers, which in turn has consequences on spectral characteristics of speech. Since MFCC characterise these spectral properties, it is reasonable to find these differing operation points for female and male models. Another interesting observation is that the systems trained with female voices generally performed worse than those trained with male data; occurring in two trials except when the SVD dataset was considered. This phenomenon has long been recognised in other speech-based applications, where a decreased performance is often achieved in systems based on spectral/cesptral characterisation and female speech [43]. Within the AVCA field, authors in [44], have also reported a reduced performance of systems based on female data. A possible explanation of this phenomenon, might be related to the differences in $f_{0}$ between male and females which has consequences in the spectral/cepstral analyses. Similarly, the presence of a glottal gap which occurs more frequently for female speakers, and which is correlated to breathiness, might be a confounding factor as well. Notwithstanding, it is difficult to conclude as there is not enough evidence supporting such behaviour and there is even a negative result about this particular.

\subsection{Experiment 3: testing out the performance of classification techniques used in speaker recognition}

The current experiment explores the usefulness of classification techniques employed in speaker recognition in labors of pathological detection. Two trials are carried out using sustained phonation and running speech of the SVD dataset. Different configurations, presented in Table 1, are followed to train the UBM-based classifiers.

\subsubsection{Trial using sustained phonation}

Three configurations are tested out by varying the type of acoustic material that is employed for training the UBM and the compensation models in the IV and PLDA schemes, allowing the examination of interesting scenarios: (i) configuration $C_{1}$, a short amount of normophonic data is employed; (ii) configuration $C_{2}$ the amount of normophonic data is increased; (iii) configuration $C_{3}$ normophonic and dysphonic registers are utilised in conjunction. The best obtained results for each one of the tested configurations are depicted in Fig. 8a.

From the obtained outcomes it can be observed that a GMMUBM model does not provide any further improvement with respect to the baseline GMM. However, when using more complex systems that stand on the idea of UBM models some subtle improvements are attained. In particular two considerations can be made. First, the PLDA provides the best efficiency in terms of AUC (0.80). Second, the best results almost always involve the use of normophonic-only registers for training UBM and compensation models (MEEI, GMar, HUPA and EUROM). Indeed, increasing the amount of normophonic material provides performance improvements as ascertained by comparing the results of configuration $C_{1}$ (which only employs one dataset of normophonic recordings) to configuration $C_{3}$ (which employs 4 dataset of normophonic registers).

\subsubsection{Trial using running speech}

The current trial employs the running speech partition of the SVD corpus, and different combinations of ancillary datasets that define five configurations that allow the examination of interesting scenarios:

- configuration $C_{1}$, normophonic and dysphonic vowels plus voiced segments extracted from running speech are used;

- configuration $C_{2}$, normophonic data of sustained phonations and normophonic vowels extracted from running speech are employed;

- configuration $C_{3}$, normophonic sentences are considered;

- configuration $C_{4}$, normophonic sentences uttered in the same language as SVD are utilised;

- configuration $C_{5}$, similar to the latter but increasing the amount of registers used for training.

The best results for each one of the configurations are presented in Fig. 8b.

Despite the best absolute results - as ascertained by the DET curves and the AUC value - are given by the simple GMM classifier, it is worth comparing the influence of the ancillary datasets in the performance of speaker recognition classification techniques. In this respect, configuration $C_{3}$ provides the best performance, indicating that the inclusion of acoustic material based on sustained 


\begin{tabular}{llllll}
\hline & Subtype & ACC & SP & SE & AUC \\
\hline & Fe. $+M a$. & $73.66 \pm 4.47$ & 0.72 & 0.70 & 0.81 \\
S.D. & Fe.: MFCC $(20)$ & $73.45 \pm 5.76$ & 0.71 & 0.75 & 0.81 \\
& $M a .: \mathrm{MFCC}(20)$ & $73.97 \pm 7.12$ & 0.69 & 0.80 & 0.85 \\
\hline S.I. & MFCC $(12)$ & $69.62 \pm 4.67$ & 0.71 & 0.77 & 0.79 \\
\hline
\end{tabular}

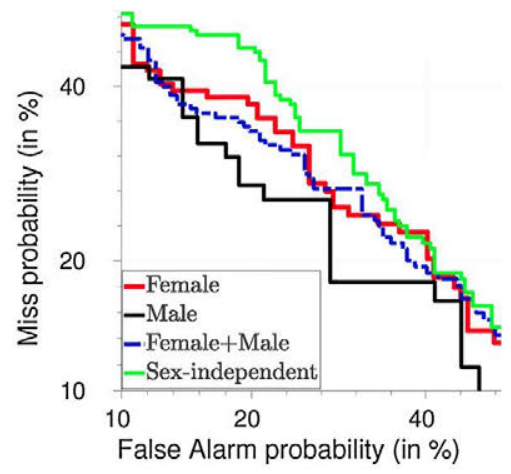

a. Results for the HUPA database.

\begin{tabular}{llllll}
\hline Type & Subtype & ACC & SP & SE & AUC \\
\hline & $F e .+M a$. & $70.74 \pm 2.27$ & 0.68 & 0.73 & 0.78 \\
S.D. & $F e .:$ MFCC $(18)$ & $72.17 \pm 2.91$ & 0.70 & 0.73 & 0.79 \\
& $M a .: \operatorname{MFCC}(16)$ & $68.68 \pm 3.62$ & 0.63 & 0.71 & 0.77 \\
\hline S.I. & MFCC $(18)$ & $70.48 \pm 2.28$ & 0.67 & 0.73 & 0.77 \\
\hline
\end{tabular}

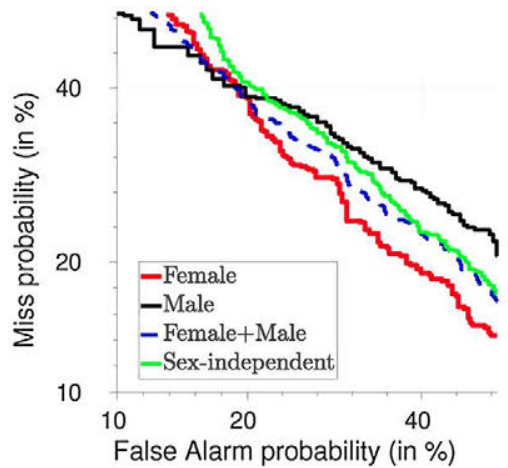

b. Results for the SVD database.

\begin{tabular}{llllll}
\hline Type & Subtype & ACC & SP & SE & AUC \\
\hline & Fe. + Ma. & $70.79 \pm 6.27$ & 0.72 & 0.70 & 0.78 \\
S.D. & $F e .:$ MFCC $(16)$ & $70.45 \pm 7.78$ & 0.69 & 0.72 & 0.77 \\
& $M a .: \operatorname{MFCC}(10)$ & $71.43 \pm 10.58$ & 0.76 & 0.66 & 0.82 \\
\hline S.I. & MFCC $(20)$ & $69.31 \pm 6.36$ & 0.69 & 0.69 & 0.77 \\
\hline
\end{tabular}

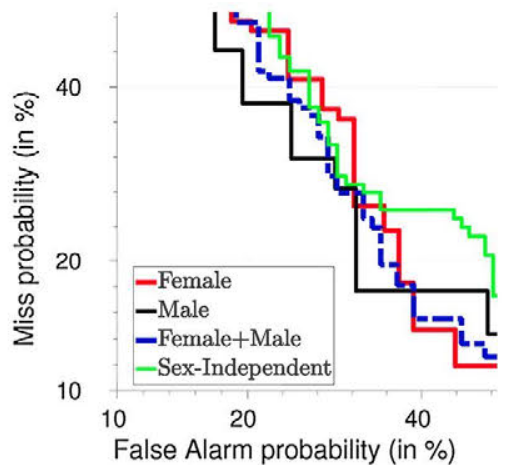

c. Results for the GMar database.

Fig. 7. Experiment 2: performance metrics and DET curves of the sex-dependent (S.D.) and sex-independent (S.I.) system using the (a) HUPA, (b) SVD and (c) GMar corpus. $\mathrm{Fe}$.:Female, Ma.:Male.

phonation does not contribute to any enhancement (see configuration $C_{2}$ ). By contrast, configuration $C_{1}$ attains, generally, the lowest AUC. This suggests that using normophonic and dysphonic registers for training the UBM and compensation models does not improve the results. Configuration $C_{4}$ is intended to test the behaviour of the system when the auxiliary dataset matches the language of the target partition, while configuration $C_{5}$ is similar to configuration $C_{4}$ but employing two auxiliary datasets. However, again, no further improvements in efficiency are found.

\subsection{Experiment 4: Combination of the best systems}

The current experiment is aimed at designing AVCA systems with the insight obtained from the previous trials. The idea is to employ the best set of features in experiment 1 , considering the hierarchical models of experiment 2 and the speaker recognition techniques of experiment 3. The acoustic material of different sources is then fused by means of logistic regression.

Following this approach, a strategy based on feature selection is followed to rank the most consistent and generalist set via MIM, mRMR, JMI and a scoring procedure of the features analysed in experiment 1 . The top-10 best characteristics for each dataset, and the top-10 global best set after having used the scoring procedure are presented in Table 2 .

Results indicate that GNE, CHNR and RALA are the most consistent features amongst datasets and speech tasks. PE and CPPS are also proficient, but in the case of the first it is just ranked in 10th position when using the GMar corpus, whereas the latter is not even 


\begin{tabular}{llllll}
\hline Configuration & Classifier & ACC & SP & SE & AUC \\
\hline- & GMM & $70.48 \pm 2.28$ & 0.67 & 0.73 & 0.77 \\
\hline \multirow{4}{*}{$C_{1}$} & GMM-UBM & $69.27 \pm 2.37$ & 0.68 & 0.70 & 0.76 \\
& IV & $67.15 \pm 2.41$ & 0.66 & 0.68 & 0.75 \\
& PLDA & $71.66 \pm 2.31$ & 0.70 & 0.73 & 0.79 \\
& GMM-SVM & $71.18 \pm 2.32$ & 0.69 & 0.73 & 0.77 \\
\hline \multirow{4}{*}{$C_{2}$} & GMM-UBM & $69.20 \pm 2.37$ & 0.69 & 0.69 & 0.76 \\
& IV & $68.38 \pm 2.38$ & 0.66 & 0.70 & 0.75 \\
& PLDA & $72.76 \pm 2.28$ & 0.72 & 0.73 & 0.79 \\
& GMM-SVM & $72.01 \pm 2.30$ & 0.69 & 0.74 & 0.77 \\
\hline \multirow{3}{*}{$C_{3}$} & GMM-UBM & $68.24 \pm 2.39$ & 0.67 & 0.69 & 0.75 \\
& IV & $70.77 \pm 2.33$ & 0.71 & 0.71 & 0.78 \\
\hline & PLDA & $71.32 \pm 2.32$ & 0.69 & 0.73 & 0.80 \\
& GMM-SVM & $71.73 \pm 2.31$ & 0.70 & 0.73 & 0.78 \\
\hline
\end{tabular}

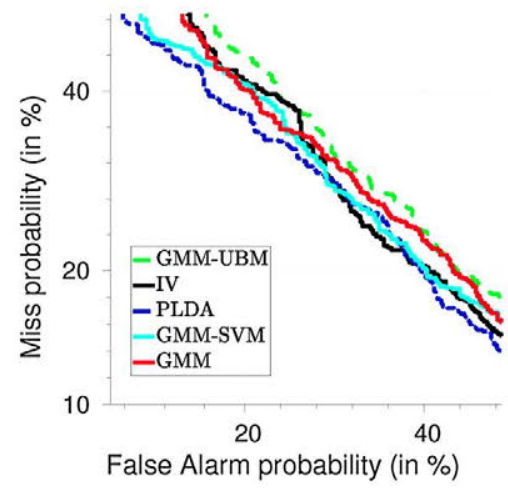

a. Trial using sustained phonation.

\begin{tabular}{|c|c|c|c|c|c|}
\hline Configuration & Classifier & $\mathrm{ACC}$ & SP & SE & AUC \\
\hline- & GMM & $80.32 \pm 2.02$ & 0.74 & 0.84 & 0.86 \\
\hline \multirow{4}{*}{$C_{1}$} & GMM-UBM & $76.70 \pm 2.15$ & 0.75 & 0.78 & 0.84 \\
\hline & IV & $71.79 \pm 2.29$ & 0.70 & 0.73 & 0.79 \\
\hline & PLDA & $74.01 \pm 2.23$ & 0.74 & 0.74 & 0.82 \\
\hline & GMM-SVM & $77.03 \pm 2.14$ & 0.75 & 0.78 & 0.85 \\
\hline \multirow{4}{*}{$C_{2}$} & GMM-UBM & $78.44 \pm 2.09$ & 0.72 & 0.82 & 0.86 \\
\hline & IV & $73.00 \pm 2.25$ & 0.71 & 0.74 & 0.81 \\
\hline & PLDA & $75.62 \pm 2.18$ & 0.75 & 0.76 & 0.85 \\
\hline & GMM-SVM & $78.17 \pm 2.10$ & 0.76 & 0.80 & 0.85 \\
\hline \multirow{4}{*}{$C_{3}$} & GMM-UBM & $78.44 \pm 2.09$ & 0.72 & 0.82 & 0.86 \\
\hline & IV & $73.00 \pm 2.25$ & 0.71 & 0.74 & 0.81 \\
\hline & PLDA & $75.62 \pm 2.18$ & 0.75 & 0.76 & 0.85 \\
\hline & GMM-SVM & $78.71 \pm 2.08$ & 0.76 & 0.80 & 0.86 \\
\hline \multirow{4}{*}{$C_{4}$} & GMM-UBM & $76.96 \pm 2.14$ & 0.74 & 0.79 & 0.84 \\
\hline & IV & $72.73 \pm 2.26$ & 0.72 & 0.73 & 0.81 \\
\hline & PLDA & $75.62 \pm 2.18$ & 0.74 & 0.76 & 0.84 \\
\hline & GMM-SVM & $77.77 \pm 2.11$ & 0.71 & 0.81 & 0.86 \\
\hline \multirow{4}{*}{$C_{5}$} & GMM-UBM & $78.64 \pm 2.08$ & 0.73 & 0.82 & 0.85 \\
\hline & IV & $70.65 \pm 2.31$ & 0.69 & 0.71 & 0.79 \\
\hline & PLDA & $76.09 \pm 2.17$ & 0.74 & 0.77 & 0.83 \\
\hline & GMM-SVM & $79.25 \pm 2.06$ & 0.76 & 0.81 & 0.86 \\
\hline
\end{tabular}

b. Trial using running speech.

Fig. 8. Experiment 3: performance of the GMM-based classifiers in the detection of pathologies using the SVD dataset: (a) for the partition of vowel /a/ and the three tested conditions; (b) for the partitions using running speech.

Table 2

Experiment 4: top-ranked features from each dataset.

\begin{tabular}{llll}
\hline HUPA & SVD & GMar & Global best \\
\hline GNE & CHNR & RALA & GNE \\
PE & GNE & GNE & CHNR \\
RALA & RALA & CPPS & RALA \\
CHNR & PE & CHNR & PE \\
MSP $_{25}$ & DFA & He & CPPS \\
LLE & He & LLE & LLE \\
CPPS & MSP95 & CIL & MSP95 \\
MSP 95 & LLE & GSampEn & DFA \\
MSH & MSH & DFA & He \\
ApEn & GSampEn & PE & CIL \\
\hline
\end{tabular}

included in the ranking of the SVD. As a result, an AVCA based solely on GNE, CHNR and RALA for characterisation and GMM classifiers to output decision scores is considered. In accordance to the results in experiment 2, the proposed systems should account for the sex of the speakers in a hierarchical-like categorisation procedure. Finally, the information of the most consistent features is fused at score level with the one provided by the UBM-based classifiers in experiment 3. Regarding the latter, and when using sustained phonation, PLDA systems trained with normophonic data are employed. For the case of running speech, the GMM algorithm is employed as it has been shown to provide better results than more complex schemes. The whole procedure is summarized graphically in Fig. 9. 


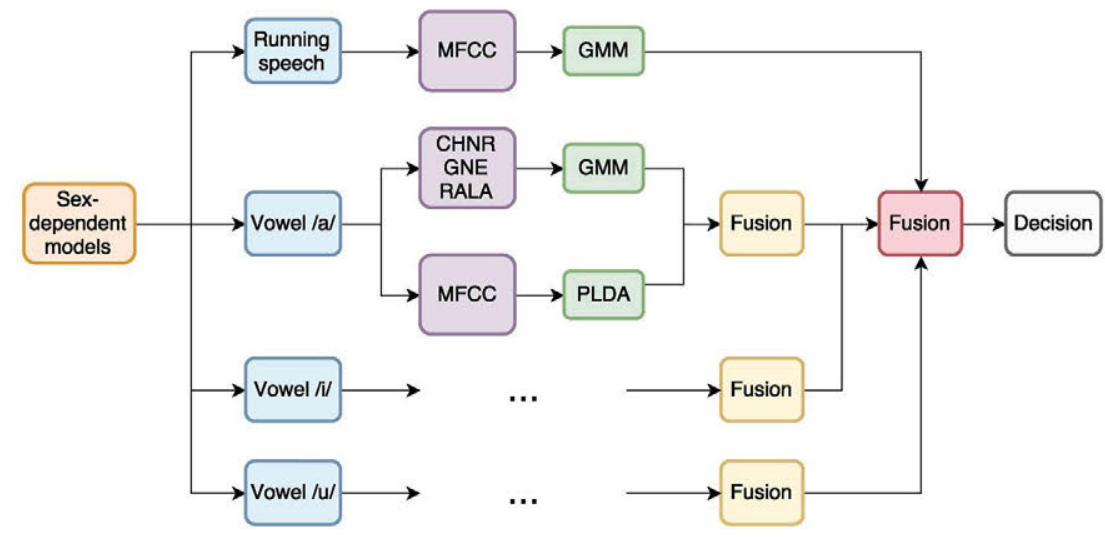

Fig. 9. Experiment 4: resulting methodology after having considered the outcomes of experiments 1, experiment 2 and experiment 3.

\subsubsection{Trial in an intra-dataset scenario}

The results after considering the methodology in Fig. 9, using the vowels /a/, /i/, /ul, and the sentence in the SvD dataset are introduced in Fig. 10a.

The outcomes indicate that the fusion of different speech tasks increases performance, achieving in the best case scenario an AUC of 0.88 and a DET curve that is better than the remaining in all operation points.

\subsubsection{Trial in a cross-dataset scenario}

The current trial is focused on training an AVCA system with data of SVD and the methodology in Fig. 9, but following a cross-dataset scenario tested in other four partitions:

- vowels /a/, /i/ and /u/ of GMar;

- the vowel /a/ in HUPA;

- registers of the vowel/a/in ATIC;

- and recordings of the vowel /a/ in DN.

The best results for all the tested partitions are presented in Fig. $10 \mathrm{~b}$.

In general terms the results are acceptable, with an AUC varying between 0.75 to 0.94 . When the vowel /a/ is considered AUC is always superior to 0.82 . In particular for the GMar corpus, no performance enhancement is obtained when all the vowels are fused.

\section{Discussion}

Results indicate that a segmentation according to the sex of the speaker improves the performance, which in absolute terms varies between $0.3 \%$ and $4 \%$ depending on the dataset. These results are in line with those found in literature, where the classification accuracy of an automatic detector of pathology is lightly improved by using a manual segmentation of the dataset according to the sex of the speakers [52]. Results suggest that partitioning the dataset according to this extralinguistic criterion improves performance, indicating the usefulness of hierarchical systems that decompose the voice pathology detection problem into smaller sub-problems. Indeed, it has been found that the best operation points for female and male systems differ. This is expected as the vocal tract and vocal folds of both sexes differ, which in turn produces subsequent changes in spectral characteristics that consequently impacts the AVCA systems. Accounting for these differences separately simplifies the detection problem and provides performance enhancements.

With respect to the trials involving different sets of features, it has been demonstrated that, as expected, no single measurement characterises entirely the phenomena related to dysphonia. This suggests that multidimensional approaches should be followed to design AVCA systems. Outcomes indicate that measures based on perturbation and cepstrum produce positive results in detection of pathologies. Indeed, as it has been ascertained during experiment 4, the most consistent features are GNE, RALA and CHNR (along with CPPS and PE). The feasibility of these features has also been extensively demonstrated by the individual classification they provide, suggesting the feasibility of using this type of features for detection purposes. Interestingly, these characteristics come from different contexts. However, a performance improvement is usually obtained when they work in conjunction, evidencing its complementarity.

In reference to the experiment 3 involving the speaker recognition classification techniques, it has been found that these classifiers have performed well when sustained phonation is used along with ancillary data of normophonic sustained phonation. By contrast, none of the datasets has contributed to enhance the performance when using running speech. In this regard, it is worth noting that the SVD dataset is composed of a single sentence that is uttered by German speakers, whereas Albayzin uses diverse sentences produced by Spanish speakers and MEEI contains registers of a text uttered in English. This mismatch might have affected the results as phonetics differs among languages. In an attempt to examine an scenario on which both ancillary and target coprora share the same language, configuration $C_{4}$ and configuration $C_{5}$ have been included. Notwithstanding, results indicate that not even under this setting the performance improved. In this particular case, we might attribute the diminished performance to the mismatch between the acoustic content of SVD, and that of EUROM and/or PhoneDat-I. A premise in the speaker recognition field, is that when the lexical content employed for the ancillary corpora matches with the one used for enrolment, a better performance is generally obtained [45]. When there is a mismatch, it is often necessary to include more data to generate more robust models that operate under a text-independent scenario. This is the reason for which the trial using sustained phonation of SVD reported positive outcomes as the ancillary dataset is composed of sustained vowels, matching with the lexical information of the SVD partition. Moreover, since sustained phonation is relatively unaffected by language this effect is minimised. Finally, it is worth to remark that a common tendency that is observed in both trials is that better results are often obtained when normophonic data is used to construct models. A hypothesis explaining this, might be related to normophonic phonation having a more compact representation in the feature space (as there does not exist variability due to pathologies), which in turn permits a better adjustment of the initialisation models of the UBM systems. It is still necessary, though, to study 


\begin{tabular}{ccccc}
\hline & ACC & SP & SE & AUC \\
\hline Vowel /a/ & $75.42 \pm 2.19$ & 0.74 & 0.76 & 0.84 \\
Vowel /i/ & $72.33 \pm 2.27$ & 0.70 & 0.73 & 0.81 \\
Vowel /u/ & $72.20 \pm 2.28$ & 0.71 & 0.73 & 0.80 \\
R.S. & $80.66 \pm 2.01$ & 0.70 & 0.86 & 0.87 \\
\hline All speech & $81.40 \pm 1.98$ & 0.71 & 0.87 & 0.88 \\
\hline
\end{tabular}

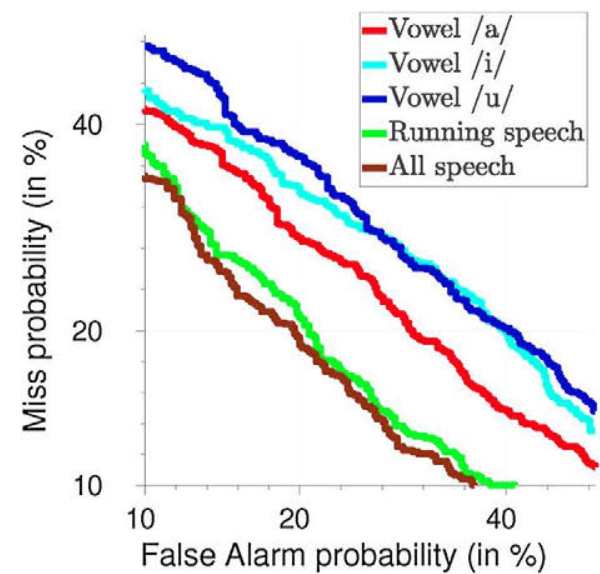

a. Results of the experiment in an intra-dataset scenario.

\begin{tabular}{lccccc}
\hline Database & Vowel & ACC & SP & SE & AUC \\
\hline & /a/ & $72.00 \pm 7.19$ & 0.60 & 0.83 & 0.82 \\
GMar & /i/ & $62.67 \pm 7.74$ & 0.47 & 0.77 & 0.75 \\
& /u/ & $72.67 \pm 7.13$ & 0.71 & 0.74 & 0.79 \\
& All & $74.00 \pm 7.02$ & 0.64 & 0.83 & 0.82 \\
\hline HUPA & $/ \mathrm{a} /$ & $74.46 \pm 4.43$ & 0.65 & 0.85 & 0.87 \\
\hline ATIC & $/ \mathrm{a} /$ & $78.21 \pm 9.16$ & 0.90 & 0.74 & 0.93 \\
\hline DN & $/ \mathrm{a} /$ & $82.87 \pm 5.49$ & 0.76 & 0.89 & 0.94 \\
\hline
\end{tabular}

b. Results of the experiment in cross-dataset scenarios.

Fig. 10. Experiment 4: Performance metrics for the trials in: (a) an intra-dataset scenario; (b) cross-dataset scenario.

the influence of dysphonic recordings in the training of compensation models. This also highlights the need of having more data available for the study of AVCA systems. Hence, translating methodologies used in speaker recognition (such as those based on UBM classifiers), should start by considering a translation of equivalent datasets to the AVCA field.

Outcomes also highlight the necessity of larger and better balanced corpora that fully describe variability factors such as age and sex, as these seem to affect AVCA systems. There is a large room for improving the systems presented in the current experiment, including a more thorough study of other types of features and classifiers correlating to ageing voices and/or to sexual conditions. In addition, other factors influencing performance should also be investigated, including accents, vocal effort, etc. An additional aspect that might be worth to consider, is in the study of methodologies that include more effectively extralinguistic information into the system, be it in the form of an a-priori probability or in a regression-like scheme.

\section{Conclusions}

The state of the art in AVCA systems reports lots of works evaluated under well controlled scenarios, providing results that suggest that the problem is almost solved. However, despite of these promising results, these systems are still far away from the clinical setting, because their accuracies are obtained in laboratory conditions and are quite dependent on the dataset used. With the aim to present more truthful results, this paper contributes with a more realistic baseline system, which can be used in the future for comparison purposes. To this respect, this paper analizes several variability factors affecting the robustness of these systems. Multiple experiments were performed to test out the influence on the performance of the speech task, extralinguistic aspects (such as sex), the acoustic features and the classifiers. The methodology followed has been developed to obtain dataset-independent results, so that they could be extrapolated to other corpora. In this sense, an analysis of several factors affecting the design of automatic voice pathology detection systems has been presented. Extralinguistics such as sex have been studied in hierarchical-like schemes, along with different sets of features in a variety of datasets containing speech material of diverse types. Moreover, speaker recognition classification techniques have been explored, fed with an acoustic material based on both sustained phonations and running speech.

Results demonstrate that including extralinguistic information regarding the sex of the speaker enhances the performance of an automatic detector of pathologies, suggesting that decomposing the pathology detection problem into sub-problems according to a certain extralinguistic criterion, decreases the complexity of the underlying models and therefore improves the efficiency of the system.

In reference to the types of speech tasks that have been evaluated, results suggest that the sustained vowel /a/ always achieve 
better results in comparison to other types of vowels. Notwithstanding, these results are outperformed when cepstral analysis and running speech is considered. Results also suggest that fusing the acoustic material of different speech tasks (vowels /a/, /i/, /u/, running speech) - via logistic regression - improves the performance of the system even further. This fusion stage has demonstrated its utility to combine information of heterogeneous but complementary systems, into a single decision machine that behaves better than the individual systems.

The speaker recognition classifiers have moderately improved results for those cases where sustained vowels are used in conjunction with ancillary corpora based on normophonic phonations. By contrast, nothing can be concluded regarding the use of running speech and these classifiers, as the phonetic mismatch between target and ancillary corpora has presumably affected the resulting UBM-based classifiers. Moreover, due to the unavailability of datasets containing dysphonic registers of running speech, an analysis on the effects of this acoustic material on the training process of UBM models remains an open issue. Despite that, it is safe to say that the results reported in this paper, involving the usage of UBM-based classifiers and running speech are not positive.

Besides, outcomes suggest that no single parameter is capable of completely characterizing vocal pathology, and therefore multidimensional approaches are needed to enhance classification results. This fact has been reported through the analysis of several sets of features and speech tasks. The most coherent features, as ascertained in several trials, are two estimators of perturbation noise and one descriptor of dispersion based on the modulation spectrum; namely, GNE, CHNR and RALA. Interestingly, the latter is a novel characteristic that has been recently introduced in $[26,38]$.

Regarding the results in numeric terms, the system trained with the SVD dataset following the approach in experiment 4 achieves an ACC of $81 \%$, which to the best of the author's knowledge one of the best and more realistic results reported in the literature for this corpus. In a cross-dataset scenario the AUC of the system reached values ranging from 0.75 to 0.94 , demonstrating that the procedures followed are robust to handle mismatches between training and testing conditions. It is worth noting that there exist in literature works reporting values superior to the ones in this paper, but many of those have only included a small portion of the dataset or have limited their analyses to a restricted number of voice pathologies. We expect that the results presented in this paper will help to establish a baseline for future comparisons following procedures that can be replicated by other researchers.

In general terms, it can be concluded that a methodology based in hierarchical detection, characterisation through a reduced set of consistent features and fusion of different speech tasks enhances the performance of the system. The cross-dataset trials have been demonstrated the robustness of the proposed AVQA system to mismatches in recording conditions, providing more realistic outcomes compared to intra-dataset experiments. As future work we plan to examine the feasibility of the system in a clinical setting, where the proposed system is evaluated and assessed as an assistive tool in the detection of pathologies. Similarly, we plan to study the influence of other extralinguistics such as age. This includes an in-depth analysis to correlate chronological to physiological age, and to establish frontiers to differentiate among age groups (young, elders, etc.). Similarly, paralinguistic information such as mood and accent are to be studied.

\section{Acknowledgements}

This work was supported by the Ministry of Economy and Competitiveness of Spain under grant DPI2017-83405-R1, and "Becas de Ayuda a la Movilidad" of the Universidad Politécnica de Madrid.

\section{References}

[1] J.B. Snow, J.J. Ballenger, Ballenger's Otorhinolaryngology: Head and Neck Surgery, 2003.

[5] S.N. Awan, N. Roy, C. Dromey, Estimating dysphonia severity in continuous speech: application of a multi-parameter spectral/cepstral model, Clin. Linguist. Phonet. 23 (11) (2009) 825-841, http://dx,doi,org/10.3109/ 02699200903242988

[6] J.I. Godino-Llorente, N. Sáenz-Lechón, V. Osma-Ruiz, S. Aguilera-Navarro, P. Gómez-Vilda, An integrated tool for the diagnosis of voice disorders, Med. Eng. Phys. 28 (3) (2006) 276-289.

[7] J.I Godino-Llorente, V. Osma-Ruiz, N. Sáenz-Lechón, I. Cobeta-Marco, R. González-Herranz, C. Ramírez-Calvo, Acoustic analysis of voice using WPCVox: a comparative study with multi dimensional voice program, Eur. Arch. Oto-Rhino-Laryngol. 265 (4) (2008) 465-476.

[8] M. Putzer, W.J. Barry, Instrumental dimensioning of normal and pathological phonation using acoustic measurements, Clin. Linguist. Phonet. 22 (6) (2008) 407-420, http://dx,doi,org/10,1080/02699200701830869.

[9] Saarbrüecken voice database. http://www.stimmdatenbank.coli.uni-saarland, de/index.php4.

[10] D. Chan, A, Fourcin, D, Gibbon, B, Grandstrom, M. Huckvale, G, Kokkinakis, K. Kvale, I. Lamel, B. Lindberg, A. Moreno, J. Mouropoulos, E, Senia, I. Trancoso, C in'T Veld, J. Zeiliger, EUROM - A Spoken Language Resource for the EU, 1995.

[11] A. Moreno, D. Poch, A. Bonafonte, E. Lleida, J. Llisterri, J.B. Mario, C. Nadeu, Albayzin speech database: design of the phonetic corpus, EUROSPEECH (1993) $175-179$

[12] C. Draxler, Introduction to the Verbmobil-Phondat database of spoken German, Proc. 3rd Int. Conf. Practical Application Prolog (1995) 201-212.

[13] Massachusetts Eye and Ear Infirmary, Voice Disorders Database, version.1.03 [cd-rom], Kay Elemetrics Corp., Lincoln Park, NJ, 1994.

[14] V. Parsa, D.G. Jamieson, Acoustic discrimination of pathological voice: sustained vowels versus continuous speech, J. Speech Lang. Hear. Res. 44 (2) (2001) 327

[15] J.B. Alonso-Hernandez, J. De Leon, I. Alonso, M.A. Ferrer, Automatic detection of pathologies in the voice by HOS based parameters, Eurasip J. Appl. Signal Process. 2001 (4)(2001) 275-284, 10.1155/\$1110865701000336.

[16] "aplicación de las tecnologías de la información y comunicaciones" database. http://www.atic.uma.es/index_atic.html.

[17] J.I. Godino-Llorente, P. Gómez-Vilda, M. Blanco-Velasco, Dimensionality reduction of a pathological voice quality assessment system based on Gaussian mixture models and short-term cepstral parameters, IEEE Trans. Biomed. Eng. 53 (10) (2006) 1943-1953.

[18] Y. Zhang, J.J. Jiang, Acoustic analyses of sustained and running voices from patients with laryngeal pathologies, J. Voice 22 (1) (2008) 1-9.

[19] H. Kasuya, Normalized noise energy as an acoustic measure to evaluate pathologic voice, J. Acoust. Soc. Am. 80 (5) (1986) 1329.

[20] G. de Krom, A cepstrum-based technique for determining a harmonics-to-noise ratio in speech signals, J. Speech Lang. Hear. Res. 36 (2) (1993) 254-266, http://dx.doi.org/10.1044/jshr.3602.254.

[21] D. Michaelis, T. Gramss, H.W. Strube, Glottal-to-noise excitation ratio: a new measure for describing pathological voices, Acta Acust. United Acust. 83 (4) (1997) 700-706.

[22] H. Hermansky, Perceptual linear predictive (PLP) analysis of speech, J. Acoust. Soc. Am. 87 (1990) 1738.

[23] J.D. Arias-Londoño, J.I. Godino-Llorente, N. Sáenz-Lechón, V. Osma-Ruiz, G, Castellanos-Domínguez, Automatic detection of pathological voices using complexity measures, noise parameters, and mel-cepstral coefficients, IEEE Trans. Biomed. Eng. 58 (2) (2011) 370-379, http://dx.doi.org/10.1109/TBME 2010.2089052.

[24] J. Hillenbrand, R.A. Houde, Acoustic correlates of breathy vocal quality: dysphonic voices and continuous speech, J. Speech Lang. Hear. Res. 39 (2) (1996) 311

[25] L. Atlas, S.A. Shamma, Joint acoustic and modulation frequency, EURASIP J. Adv. Signal Process. 2003 (7) (2003) 310290.

[26] L. Moro-Velázquez, J.A. Gómez-García, J.I. Godino-Llorente, G. Andrade-Miranda, Modulation spectra morphological parameters: a new method to assess voice pathologies according to the GRBAS scale, Biomed. Res. Int. (2015).

[27] H. Kantz, T. Schreiber, Nonlinear Time Series Analysis, 2nd ed., Cambridge University Press, 2004.

[28] M.A. Little, P.E. McSharry, S.J. Roberts, D.A. Costello, I.M. Moroz, Exploiting nonlinear recurrence and fractal scaling properties for voice disorder detection, Biomed. Eng. Online 6 (1) (2007) 23, http://dx.doi,org/10.1186/ $1475-925 X-6-23$.

[29] C. Peng, S. Havlin, H.E. Stanley, A.L. Goldberger, Quantification of scaling exponents and crossover phenomena in nonstationary heartbeat time series, Chaos Interdiscip. J. Nonlinear Sci. 5 (1) (1995) 82-87.

[30] S.M. Pincus, Approximate entropy as a measure of system complexity, Proc. Natl. Acad. Sci. U.S.A. 88 (6) (1991) 2297-2301, http://dx.doi.org/10.1073/ pnas.88.6.2297.

[31] J.S. Richman, J.R. Moorman, Physiological time-series analysis using approximate entropy and sample entropy, Am. J. Physiol. Heart Circ. Physiol. 278 (6) (2000) H2039-H2049.

[32] H.-B. Xie, W.-X. He, H. Liu, Measuring time series regularity using nonlinear similarity-based sample entropy, Phys. Lett. A 372 (48) (2008) 7140-7146. 
[33] L. Xu, K. Wang, L. Wang, Gaussian kernel approximate entropy algorithm for analyzing irregularity of time-series, Proceedings of 2005 International
Conference on Machine Learning and Cybernetics (2005) 5605-5608.

[34] W. Chen, Z. Wang, H.-B. Xie, W. Yu, Characterization of surface EMG signal based on fuzzy entropy, IEEE Trans. Neural Syst. Rehabil. Eng. 15 (2) (2007) 266-272, http://dx.doi.org/10.1109/TNSRE.2007.897025.

[35] C. Bandt, Ordinal time series analysis, Ecol. Model 182 (3-4) (2005) 229-238.

[36] M. Zanin, L. Zunino, O.A. Rosso, D. Papo, Permutation entropy and its main biomedical and econophysics applications: a review, Entropy 14(12)(2012) 1553-1577, http://dx,doi.org/10,3390/e14081553.

[37] J.D. Arias-Londoño, J.I. Godino-Llorente, Entropies from Markov Models as complexity measures of embedded attractors, Entropy 17 (6) (2015) 3595-3620, http://dx.doi.org/10.3390/e17063595.

[38] L. Moro-Velázquez, J.A. Gómez-García, J.I. Godino-Llorente, Voice pathology detection using modulation spectrum-optimized metrics, Front. Bioeng. Biotechnol. 4 (1) (2016).

[40] M. Cord, P. Cunningham (Eds.), Machine Learning Techniques for Multimedia, Cognitive Technologies, Springer Berlin Heidelberg, Berlin, Heidelberg, 2008, http://dx.doi.org/10.1007/978-3-540-75171-7.

[41] G. Brown, A. Pocock, M.-J. Zhao, M. Luján, Conditional likelihood maximisation: a unifying framework for information theoretic feature selection, J. Mach. Learn. Res. 13 (2012) 27-66.

[42] F. Schiel, Automatic phonetic transcription of non-prompted speech, Proc, of the ICPhS, San Francisco (1999) 607-610.

[43] J. Mason, J. Thompson, Gender effects in speaker recognition, in: Proc. ICSP-93, Beijing, 1993, pp. 733-736.
[44] R. Fraile, N. Sáenz-Lechón, J.I. Godino-Llorente, V. Osma-Ruiz, C. Fredouille, Automatic detection of laryngeal pathologies in records of sustained vowels by means of MEL-frequency cepstral coefficient parameters and differentiation of patients by sex, Folia Phoniatr. Logop. 61 (3) (2009) 146-152, http://dx.doi.org/10.1159/000219950.

[45] A. Larcher, K.A. Lee, B. Ma, H. Li, Text-dependent speaker verification: classifiers, databases and RSR2015, Speech Commun. 60 (2014) 56-77.

[52] J.A. Gómez-García, I. Moro-Velázquez, J.I. Godino-Llorente, C.G.

Castellanos-Dominguez, An insight to the automatic categorization of speakers according to sex and its application to the detection of voice pathologies: a comparative study, Revista Facultad de Ingeniería 79 (2016) $50-62$.

[53] W.M. Campbell, D.E. Sturim, D.A. Reynolds, Support vector machines using GMM supervectors for speaker verification, IEEE Signal Process. Lett. 13(5) (2006) 308-311.

[54] Brookes, Mike. VOICEBOX: Speech Processing Toolbox for MATLAB, 2005. Available online: www.ee.ic.ac.uk/hp/staff/dmb/voicebox/voicebox.html.

[55] N. Dehak, P.J. Kenny, R. Dehak, P. Dumouchel, P. Ouellet, Front-end factor analysis for speaker verification, IEEE Trans. Audio Speech Lang. Process. 19 (4) (2011) 788-798.

[56] D. Garcia-Romero, C.Y. Espy-Wilson, Analysis of i-vector length normalization in speaker recognition systems, In Twelfth Annual Conference of the International Speech Communication Association (2011)

[57] D.A. Reynolds, T.F. Quatieri, R.B. Dunn, Speaker verification using adapted Gaussian mixture models, Digital Signal Process. $10(1-3)(2000)$ 19-41. 